\title{
Cem anos de historiografia da Primeira Guerra Mundial: entre história transnacional e política nacional*
}

\author{
Sílvia Adriana Barbosa Correia**
}

\section{RESUMO}

Aproveitando a rememoração do centenário do início da Primeira Guerra Mundial, procura-se aqui delinear uma incursão breve na forma como foi e tem sido lida a Primeira Guerra Mundial pela disciplina histórica. Pretende-se ressaltar as tendências historiográficas mais determinantes nos cem anos posteriores ao fim do conflito nos principais centros acadêmicos e referir alguns dos trabalhos mais recentes em torno das questóes que pautaram desde sempre o debate em torno do conflito.

Palavras-chave: Primeira Guerra Mundial; historiografia; centenário.

\section{ABSTRACT}

At the centenary of the World War I outbreak, this paper seeks to outline a brief overview into the way WWI has since been read by the historiography. It intends to emphasize the most crucial historiographical trends in the hundred years after the beginning of the conflict in the leading academic centers, and point out some of the more recent work concerning the issues that have always guided the debate over the conflict.

Keywords: World War I; historiography; centenary.

Ensaio - DOI - http://dx.doi.org/10.1590/2237-101X015029011

Ensaio bibliográfico recebido em 1ํ de setembro de 2014 e aceito em 20 de setembro de 2014.

* Texto adaptado do primeiro capítulo de CORREIA, S. Politicas da memória da I Guerra Mundial em Portugal, 1918-1933. 2011. 523 f. Tese (Doutorado em História) — Faculdade de Ciências Sociais e Humanas, Universidade Nova de Lisboa, Lisboa, 2011.

** Doutora em História pela Universidade Nova de Lisboa, e professora adjunta da Universidade Federal do Rio de Janeiro (UFRJ). Rio de Janeiro, RJ, Brasil. Email: sabcorreia@gmail.com. 
A experiência fundamental da guerra moderna é, não sem alguma perturbação, "o assassinato de massa sancionado pelo Estado". ${ }^{1}$ Expressóes como esta denunciam sem dúvida a profundidade material e imaterial do impacto do conflito em questão. Incontrolavelmente, um acontecimento desta natureza iria inevitavelmente transformar a história e a historiografia. A questão que esta revisão bibliográfica irá conduzir — ainda que não ambicione esgotá-la - é a de como, ao longo de cem anos, a Primeira Guerra Mundial foi lida. Pretende-se ressaltar as tendências historiográficas mais determinantes nos cem anos posteriores ao fim do conflito, nos principais centros acadêmicos, e referir alguns dos trabalhos mais recentes a respeito das questóes que pautaram, desde sempre, o debate em torno do conflito.

Parte-se, porém, do pressuposto de que o exercício historiográfico é uma importante acumulação de incursôes e temáticas que ontem e hoje transformaram a análise da Primeira Guerra Mundial num dos campos percursores da história do século XX. Não obstante a resistente persistência de uma dimensão nacional nas políticas oficiais rememorativas que assinalam o centenário, não mais a historiografia se desligará de uma perspectiva transnacional. Decorre esta nevrálgica viragem de uma coexistente maturação da história política, social e econômica, mas essencialmente cultural, que traria para o palco da guerra sujeitos que não mais poderiam ser pensados apenas no âmbito de um tempo e espaço vedados pelas fronteiras do nacional.

A historiografia da Grande Guerra acompanha, com algum protagonismo, as mudanças operadas na historiografia do século XX ao longo de quatro fases, que não são estanques. A amplitude do trabalho em torno da guerra leva à possibilidade de múltiplos confrontos entre geraçôes ou mesmo entre pesquisadores na mesma geração de forma dialógica. ${ }^{2}$ Porém, apesar de, como reflete Jay Winter, o trabalho dos historiadores ser atualmente marcado por uma prática de uma quarta geração, não inibe que se preste atenção aos trabalhos anteriores, já que a historiografia da Grande Guerra é marcada por fortes continuidades.

A análise mais consistente da evolução da historiografia da Grande Guerra deve-se a Antoine Prost e Jay Winter em Penser la Grande Guerre, publicado em 2004. ${ }^{3}$ Esses autores procuraram ultrapassar os limites nacionais e delinear o processo evolutivo (geral) da historiografia. ${ }^{4}$ Considerando três fases, defendem que a transição da primeira para a segunda linha historiográfica é de ruptura, enquanto da segunda para a terceira é de continuidade evolutiva. É interessante verificar que alguns autores assumem essa transição mais fluida nos

\footnotetext{
${ }^{1}$ MOSSE, George L. Le guerre mondiali dalla tragedia al mito dei caduti. Roma: Laterza, 1990. p. 3.

2 WINTER, Jay (Ed.). The Legacy of the Great War: Ninety Years On. Columbia; Londres: University of Missouri Press, 2009. p. 1.

${ }^{3}$ WINTER, Jay; PROST, Antoine. Penser la Grande Guerre: Un essai d'historiographie. Paris: Éditions du Seuil, 2004. p. 15-50.

${ }^{4}$ Nomeadamente, fazem uma análise mais alargada das questôes históricas relativas ao debate em torno deste conflito, visando não apenas os trabalhos de historiadores, mas também de outras áreas científicas importantes, com especial atenção para a literatura anglófona, alemã traduzida para inglês, e italiana (excluindo a russa).
} 
seus próprios trabalhos, como é o caso, entre outros, de Jay Winter e de Jean-Jacques Becker. Porém, o próprio Jay Winter, numa das suas mais recentes obras e apresentaçôes públicas, identifica uma quarta geração historiográfica que, como aqui ficará evidente, é mais uma novidade, não de ruptura, mas de continuidade no trabalho de pesquisa que vinha sendo desenvolvido em torno da Primeira Guerra Mundial. ${ }^{5}$

Vejamos, num primeiro momento, como se materializam essas configuraçôes históricas em torno da Grande Guerra, dando, numa segunda fase, atenção à forma como essas transformaçóes se concretizam em algumas das mais recentes pesquisas.

\section{Configurações historiográficas}

A primeira geraçáo é contemporânea ao conflito, apelidada the Great War generation (a geração da Grande Guerra). Entendendo a excepcionalidade do momento, ela é orientada por políticos, soldados e oficiais que experienciavam ou haviam experienciado a guerra, pronunciando discursos de propaganda para posterior apuramento de responsabilidade. Daí os textos de Pierre Renouvin — Les origines immédiates de la guerre (1925) ou La crise Européenne et la Grande Guerre (1934) — ou de Jules Isaac — Un débat historique, le probléme des origines de la guerre (1931). O jovem historiador Pierre Renouvin, ainda no calor político do imediato pós-guerra, a partir de uma abordagem événementielle, que logo seria criticada pela escola dos Annales, e de uma súmula de documentos que alguns governos publicaram em torno da responsabilidade da guerra, procura desconstruir a ideia de que as potências centrais teriam total responsabilidade na eclosão da Grande Guerra. Argumento que outros autores irão de certa forma completar, como o fez Jules Isaac em 1933, embora com algumas variaçóes. ${ }^{6}$

Numa narrativa contada de cima para baixo, focavam a análise no Estado como ator central no esforço de guerra. Outros, num tom autojustificativo, sejam generais, políticos ou veteranos, escrevem no sentido de repor o seu lugar na vitória ou pedagogicamente apresentar algumas liçôes a ser apreendidas face a um novo conflito. Porém, tratavam-se de trabalhos densos e técnicos que raramente teriam aplicabilidade, dada a pouca divulgaçáo, em experiências de guerra mais próximas.

A segunda fase, entre as décadas de 1920 e 1960, é dominada por uma história diplomática e militar indiferente às temáticas mais ligadas aos combatentes, sendo rara a publicação de monografias que os analise. Nessa época, a universidade é ainda uma instituição peque-

\footnotetext{
${ }^{5}$ WINTER, Jay (Ed.). The Legacy of the Great War: Ninety Years On, op. cit. (publicado no $90^{\circ}$ aniversário da Guerra) e WINTER, Jay. “The Great War in Transnational Perspective”, in: World War One International Conference. Londres: Queen Mary University of London, 1-4 de ago. 2014.

${ }^{6}$ PROST, Antoine. 1925: Renouvin et les origines de la première guerre mondiale. Le Monde, 4 nov. 2013. Disponível em: <http://www.lemonde.fr/livres/article/2013/11/04/1925-renouvin-et-les-origines-de-la-premiere-guerre-mondiale_3507594_3260.html>. Acesso em: 14 set. 2014.
} 
na. Apesar do reconhecimento conferido à disciplina de história, esta não possuía o contingente necessário de professores para produzir um discurso com suficiente repercussão nos debates sobre a guerra. A primeira expressão desses estudos será da responsabilidade da ainda existente Bibliothèque de Documentation Internationale Contemporaine (BDIC), criada, em 1918, com o fim de compreender as causas da Primeira Guerra Mundial e de congregar todo o material disponível sobre esse e outros acontecimentos do tempo presente. Paralelamente, apela-se ao pacifismo através da divulgação de documentos diplomáticos em torno das causas e consequências da guerra. Porém, não só se faria a história da política ou das decisóes políticas, mas também surge gradualmente a história da sociedade, "definida como a história das estruturas sociais e dos movimentos sociais"?

Nos anos 1960, irrompe a análise social da guerra, marcada a um tempo por um retorno e uma chegada. Retornam os protagonistas — os combatentes —, que gradualmente iam perecendo, e chegam novos autores que adquirem a legitimidade da escrita da história da guerra. Favorecida pelas mudanças sociais e pela afirmação do paradigma marxista, reorienta-se a história militar e política, apontando-se a guerra como resultado do imperialismo. Isso se faz pela descoberta efetiva dos combatentes. Esta valorização, ideológica e social, desde o movimento operário ao poder dos grupos sociais, pode ser balizada, entre outros, por trabalhos como os de Jonh Horne e Jay Winter (sobre o socialismo e a guerra no Reino Unido), o de Gui Pedroncini (sobre as rebelióes no exército francês - o primeiro trabalho sobre a moral das tropas, 1967 e 1968), o de Jean-Jacques Becker (interessado na mobilização dos soldados) o de Antoine Prost (dedicado aos antigos combatentes e à exclusão social dos mesmos). ${ }^{8}$ James Joll, um acadêmico da II Internacional, faria uma revisão da história diplomática sobre a Crise de Julho, ${ }^{9}$ analisando o que chama de "unsoken assumptions", na medida em que motivos menos óbvios, como código de honra, haviam movido os homens para a guerra, impedindo que se refreassem (1968). A história das mentalidades havia, segundo Prost e Winter, atravessado o Canal da Mancha e penetrado a London School of Economics.

Os anos 1960 trazem consigo uma profunda mudança na dimensão e natureza de compreensão do conflito. No âmbito da história social feita à época, desperta uma certa nostalgia de um mundo que a guerra havia destruído, um mundo que "havia sido traído por uma elite capaz de sacrificar as massas em nome de desejos táo vãos quanto glória e honra". ${ }^{10}$ Esses

\footnotetext{
${ }^{7}$ WINTER, Jay (Ed.). The Legacy of the Great War: Ninety Years On, op. cit., p. 2.

${ }^{8}$ HORNE, John. Labour at War: France and Britain 1914-1918. Oxford: Clarendon Press, 1991.

WINTER, Jay. Socialism and the Challenge of War. Ideas and Politics in Britain 1912-18. Londres: Routledge, 1974; BECKER, Jean-Jacques. 1914: Comment les Français sont entrés dans la guerre: contribution à l'étude de l'opinion publique printemps-été 1914. Paris: Presses de la Fondation Nationale des Sciences Politiques, 1977; PROST, Antoine. Les Anciens combattants et la société française, 1914-1939. 3 v. Paris: Presses de la Fondation Nationale des Sciences Politiques, 1977; JOLL, James. 1914: The Unspoken Assumptions. Londres: Weidenfeld and Nicolson, 1968.

${ }^{9}$ Conjunto dos acontecimentos que haveria de precipitar a eclosão da guerra.

${ }^{10}$ WINTER, Jay (Ed.). The Legacy of the Great War: Ninety Years On, op. cit., p. 4.
} 
trabalhos permitiram relançar o "entusiasmo" pela Primeira Guerra Mundial, entretanto perdido para a Segunda, sob a forma de uma história das mentalidades que evolui rapidamente para uma história cultural.

Definitivamente, a história da Primeira Guerra Mundial entra nas universidades e nas casas de família. Por um lado, os pesquisadores passam a ter acesso a um conjunto de fontes oficiais, disponíveis passados cinquenta anos do acontecido, abrindo todo um novo debate sobre o conflito. Por outro lado, aflora uma história pública. Depois da Segunda Guerra Mundial, a televisão entraria no quotidiano, atraindo audiências para narrativas históricas em torno da guerra a serem integradas na história de vida daqueles que participaram da guerra ou daqueles que haviam perdido os seus na frente.

A terceira geração é chamada por Jay Winter de Vietnam generation (geração Vietnã). Entre os anos 1970 e 1980, os efeitos da guerra do Vietnã, da détente (distensão) nuclear e da crise do petróleo de 1973 haviam de levar a uma contestação geral contra as intervençôes militares no mundo. A nova geração não mais veria a "just war" como algo razoável, mas sim entendida como irracional e aportando consequências catastróficas para vencedores e vencidos.

Com efeito, nos anos 1980, surgem trabalhos de investigação centrais para a História Cultural e para a memória de guerra. Na sequência de dois colóquios organizados na França, nomeadamente Les sociétés européennes et la guerre de 1914-1918 (Nanterre, 1988) e Guerre et cultures (Historial de Péronne, 1992), aparece e esclarece-se a noção de "cultura de guerra". Annette Becker e Audoin-Rouzeau, referindo-se ao primeiro conflito mundial, a definirão como "um conjunto de práticas, de representaçôes, de atitudes, de criações dos anos de 19141918. E também dos anos seguintes, tanto é verdade que este tipo de história [cultural] dá um largo espaço à recordação e à comemoração do pós-guerra". ${ }^{11}$

Convirá, sobre este ponto, acrescentar que a história dessa cultura de guerra não se confunde com a história propriamente dita da criação cultural, literária ou artística, nem daqueles que criam essas formas. Assim, como sugere Antoine Prost, a história cultural recobre a "evolução das representaçôes sociais, mas apenas como traços, entre outros, de representaçôes que lhe dâo forma e significado". ${ }^{12}$

Aliás, precisemos, nesse quadro, um anúncio que, já em 1969, George L. Mosse havia feito: "Chegou o tempo de ir além das elites e de dirigir-se no sentido de uma investigação

${ }^{11}$ Citado por LEMOINE, Thierry. 'Culture(s) de guerre', évolution d'un concept. In: LEMOINE, Thierry. Questions d'histoire contemporaine: conflits, mémoires et identités. Paris: PUF, 2006. p. 136: "l'ensemble des pratiques, des représentations, des attitudes, des créations des années 1914-1918. Et aussi des années suivantes, tant il est vrai que ce type d'histoire fait une large place au souvenir et à la commémoration de l'après-guerre". (Tradução da autora.)

${ }^{12}$ PROST, Antoine. Republican Identities in War and Peace: Representations of France in the Nineteenth and Twentieth Centuries. Oxford: Berg, 2002. p. 96: "I take cultural history not as the history of cultural, literary or artistic creation, nor as the history of those who create these forms. I take cultural history to be the evolution of social representations, but only as traces, among others, of representations that give them form and meaning" (tradução da autora). 
em profundidade das práticas populares e dos sentimentos. Numa época de política de massa e de cultura de massa, o historiador dos fenômenos intelectuais tem necessidade de novas abordagens suscetíveis de ter em conta estes aspectos populares que desempenharam um papel capital na evolução dos homens e da sociedade?”. ${ }^{13}$

Um conjunto de mudanças proporciona e contribui para a renovação das abordagens historiográficas à guerra. Insiste-se que, em grande parte, elas são devedoras de um espectro de alteraçóes dos paradigmas explicativos. No caso, talvez a principal mudança tenha a ver com a diluição progressiva do marxismo como matriz referencial. Ainda assim, o acento mais decisivo talvez seja não propriamente a mudança de explicação, mas a mudança de problemática. O plano das interrogaçóes passa a incidir sobre aspectos particulares da sociedade e não sobre o todo global. Dir-se-ia ocorrer aqui uma evolução homóloga à emergência das análises micro-históricas na historiografia de finais dos anos 1970 e durante os anos 1980.

No contexto temático da Grande Guerra, o desaparecimento daqueles que experienciaram diretamente a guerra introduz uma encruzilhada específica, gerando mudanças nos processos rememorativos. Aumenta a autoridade da História, enquanto resgate memorial. Daí, a atenção aos testemunhos, objeto de um interesse diferente: recuperar a subjetividade. Num capítulo de uma série televisiva de 1988, Jay Winter explora a memória da guerra, retomando uma via aberta por Marc Ferro. Insere esse trabalho na história cultural — uma projeção da "totalidade da guerra", afastando-se claramente da história militar, mas não totalmente da social.

A rapidez da transição entre uma segunda e uma terceira geração historiográfica explica-se pela continuidade: já existia história cultural na história social, nomeada de "opinião", "mentalidade", "psicologia”, entre outros termos. É significativo que o "avanço" da história cultural da guerra, num contexto de deslegitimação das grandes teorias, seja protagonizado por uma historiografia americana pouco impregnada pelo marxismo.

Veja-se o exemplo de Paul Fussell com The Great War and Modern Memory (1975), de John Keegan com The Face of the Battle (1977) ou No Man's Land: Combat and Identity in World War I (1979) de Eric Leed, interessando-se pelos soldados, mas num sentido mais antropológico. ${ }^{14}$ Paul Fussell procurou, acima de tudo, entender como os soldados integrariam a experiência do conflito, já que a linguagem romântica do voluntarismo heroico da

\footnotetext{
${ }^{13}$ Citado por AUDOIN-ROUZEAU, Stéphane. Préface. In: AUDOIN-ROUZEAU, Stéphane. De la Grande Guerre au totalitarisme. La brutalisation des sociétés européenes. Paris: Hachete Littératures, 1999. p. VI-VII: "Le temps est venu d'aller au-delà des élites et de se diriger vers une investigation en profondeur des pratiques populaires et des sentiments. A une époque de politique de masse et de culture de masse, l'historien des phénomènes intellectuels a besoin de nouvelles approches susceptibles de prendre en compte ces aspects populaires qui ont joué un rôle si capital dans l'évolution des hommes et à la société?" (tradução da autora). ${ }^{14}$ WINTER, Jay; PROST, Antoine. Penser la Grande Guerre: Un essai d'historiographie, op. cit., p. 42-50; FUSSELL, Paul. The Great War and Modern Memory. Nova York; Londres: Oxford University Press, 1975; KEEGAN, John. The Face of the Battle. Londres: Hutchinson, 1998; LEED, Eric J. No Man's Land: Combat and Identity in World War I. Cambridge: Cambridge University Press, 1979.
} 
propaganda de guerra se tornaria intolerável depois de vivida a violência das trincheiras. John Keegan, analisando uma série de batalhas, demonstra como o processo de industrialização — diria totalização — da guerra condicionaria a impossibilidade dos combatentes se desligarem, em suas vidas, dessa experiência. Apesar de ser um historiador militar, analisou o impacto humano e psicológico da guerra e a sua autoridade técnica levou a que este praticamente abrisse uma nova linha na história militar da guerra. Eric Leed, enfim, desmontando o universo emocional dos soldados das trincheiras, afirma que a sua permanência num estado de estresse pós-traumático os empurraria definitivamente, num pós-guerra, para movimentos milicianos extremistas.

Os trabalhos de Fussel, Keegan e Leed ajudaram, segundo Winter, a criar uma interpretação trágica da Grande Guerra. Redefiniram-na como um acontecimento que transformou a linguagem, quebrou barreiras entre esfera pública e privada, violou a distinção entre alvos civis e militares, desafiou divisóes de gênero e abriu uma nova fase na história de guerra.

Neste horizonte, é possível destacar dois movimentos na história cultural da Primeira Guerra Mundial. Um dos movimentos é protagonizado por Mario Isnenghi (1970) e George Lachman Mosse (1990), que invocam, num exercício de cultura política, o mito da experiência de guerra na base da ruptura e radicalização política das sociedades. ${ }^{15}$

$\mathrm{O}$ outro movimento parte de dois linguistas anglo-saxônicos, sem contato entre si, que encontram na memória a base da história cultural da guerra. Paul Fussell, na obra já referida, numa reação à Guerra do Vietnã, explora o absurdo da guerra, pela própria experiência que teve na Segunda Guerra Mundial, e Samuel Hynes, menos influenciado pela experiência pessoal, em La guerre imaginée (1992) e Le conte du soldat (1997) estuda a forma como a guerra cria mitos, e como os soldados procuram dar lógica à sua experiência. ${ }^{16}$ Esses autores marcariam os futuros exercícios em torno da guerra, sugerindo que a Grande Guerra constitui uma ruptura fundamental na era moderna, incompreensível precisamente sem a intensificação dos pontos de vista da história cultural.

Reconhecendo os contributos desses movimentos, Modris Eksteins vê na guerra um "presságio do modernismo"; enquanto Jay Winter institui uma perspectiva diferente, também forte, demonstrando a dominância de uma linguagem tradicional (clássica, cristã e romântica) de encarar a guerra e o luto. ${ }^{17}$

O puzzle criado pela multiplicidade das abordagens resultantes da viragem cultural da historiografia da guerra demonstra uma variedade grande de objetos, como os seguintes: guerra e loucura; relação entre civis e soldados; a guerra imaginada, a guerra representada e

\footnotetext{
${ }^{15}$ ISNENGHI, Mario. Il mito della Grande Guerra: da Marinetti a Malaparte. Bari: Laterza, 1970.

${ }^{16}$ HYNES, Samuel. The Soldier's Tale. Bearing Witness to Modern War. Nova York: Penguin Press, 1997; HYNES, Samuel. A War Imagined. The Great War and English Culture. Londres: Bodley Head, 1991.

${ }^{17}$ EKSTEINS, Modris, Rites of spring: the Great War and the birth of modern age. Boston: Houghton, 1989; WINTER, Jay. Sites of war, sites of mourning: The Great War in the European Cultural History. Cambridge: Press Syndicate of the University of Cambridge, 1995.
} 
a guerra recordada e comemorada; a realidade da retaguarda; o mundo dos civis e a sua interpretação do conflito; as razóes da guerra e os sentimentos dos entrincheirados; a violência de guerra e as práticas de consentimento. ${ }^{18}$

A diversidade de interpretações não alimenta, à partida, uma incompatibilização radical dos resultados, o que não deixa de criar uma certa perplexidade. A sobreposição das perspectivas aceita uma continuidade aparentemente lógica na abertura dos temas, sob a pressão da experiência do testemunho. É caso para perguntar se este puzzle da história cultural não responde a uma procura da sociedade contemporânea, permitindo aos historiadores um contato com os fenômenos mais íntimos e variados da existência humana. ${ }^{19}$ Antoine Prost e Jay Winter, eles próprios, protagonizam essa transição teórica, sintetizando e aglutinando divisóes interpretativas da guerra, mostrando que história cultural "é uma história de práticas significantes: ela estuda como os homens e as mulheres conferiram sentido ao mundo no qual vivem. Donde a importância do luto (...), da brutalização das sociedades (...), da violência de guerra”. Longe de qualquer simplificação abusiva, Prost e Winter resumem o caminho historiográfico feito até este momento da seguinte forma: "A primeira configuração explica a história pelas decisóes dos atores; a segunda pelo jogo das forças sociais; a última faz da cultura o motor da história e encontra nela as suas explicaçôes. As representaçôes determinam os atos." 20

\section{Incursões temáticas}

Em todos os países, principalmente naqueles que participaram da guerra, muito se tem discutido a respeito dela. Porém, existe uma clara confluência não só nas formas de se fazer essa história, mas no que diz respeito ao seu conteúdo.

\footnotetext{
${ }^{18}$ São exemplo disso, primeiro, os trabalhos de Antoine Prost e Kenneth Inglis e, depois, os de Annette Becker, Stéphane Audoin-Rouzeau, Petzold, Mark Connelly, Adrian Gregory, Alex King, entre outros. Veja-se WINTER, Jay; PROST, Antoine. Penser la Grande Guerre: Un essai d'historiographie, op. cit., p. 251-252. ${ }^{19}$ Este estudo mais intimista deve muito aos trabalhos desenvolvidos, em conjunto ou individualmente, por Annette Becker e Stéphane Audoin-Rouzeau. Por exemplo, BECKER, Annette; AUDOIN-ROUZEAU, Stéphane. 14-18 Retrouver la guerre. Paris: Gallimard, 2000; BECKER, Annette. War and Faith: the Religious Imagination in France, 1914-1930. Oxford; Nova York: Berg, 1998; BECKER, Annette. La guerre et la foi : De la mort à la mémoire, 1914-1930. Paris: A. Colin, 1994; BECKER, Annette. Les monuments aux morts : Mémoire de la Grande Guerre. Paris: Éditions Errance, 1991.

${ }^{20}$ WINTER, Jay; PROST, Antoine. Penser la Grande Guerre: Un essai d'historiographie, op. cit., p. 47-48: "C'est une histoire des pratiques signifiants : elle étudie comment les hommes et les femmes ont conféré un sens au monde dans lequel ils vivaient. D'où l'importance du deuil (WINTER, 1995; AUDOUIN-ROUZEAU, 2001), de la brutalisation des sociétés (MOSSE, 1990), de la violence de guerre"; "Mas cette histoire culturelle ne se limite pas à étendre l'investigation. Elle propose aussi d'autres paradigmes. La première configuration expliquait l'histoire par les décisions des acteurs; la deuxième par l'enjeu des forces sociales; la dernière fait de la culture de moteur de l'histoire et trouve en elle ses explications. Les représentations déterminent les actes" (tradução da autora).
} 
Jay Winter observou, na conferência que proferiu no momento em que se concretizavam cem anos do início do conflito, como poucos foram os campos historiográficos que enriqueceram assim nos últimos anos, principalmente desde início dos anos $1990 .{ }^{21}$ Depois de uma importante viragem cultural e interdisciplinar, uma incursão comparativa e transnacional dominaria os primeiros anos do século XXI, não só trazendo novos temas para o centro do debate, mas também promovendo novas incursôes em alguns dos temas mais tradicionais.

A mudança no tipo de abordagem haveria de condicionar profundamente o conteúdo da produçáo historiográfica, transferindo-se da análise das personagens, numa história militar, política e diplomática, para uma compreensão múltipla das representaçôes e da cultura popular. Ou seja, a forma como se faz história haveria de condicionar os objetos dessa mesma história.

Apesar da importante multiplicidade de abordagens e temas, importa ressalvar que ainda são alguns os espaços a preencher pela pesquisa num momento em que se consagram 100 anos do início do conflito. Tratam-se, porém, de debates e questóes que a abordagem cultural, em última instância, impulsionou, o que fez com que enfoques biográficos, econômicos, entre outros, fossem relegados para segundo plano. Por outro lado, Jay Winter, numa entrevista dada ao Público, jornal português de tiragem nacional, em julho de 2014, refere que a história cultural da guerra na frente Leste e no Império Otomano só agora começou a ser escrita. ${ }^{22}$ Ainda é o caso das recentes incursóes culturais sobre tropas e trabalhadores não brancos, procurando demonstrar de que forma uma linguagem "antropológica" e imperial passa a ser usada em território europeu para representar o inimigo. Andrew Evans (2010) mostra como os alemães categorizavam racialmente os seus prisioneiros de guerra $\mathrm{e}$ Santanu Das (2011) procura compreender como ideias de raça e colonialismo percorreriam todo o conflito. ${ }^{23}$ Quanto ao particular esforço das tropas negras, destaque-se os trabalhos de Richard S. Fogarty $(2008)^{24}$ que expóe as tensôes entre as concepçôes de república, ideologia colonial e raça na França, fenômeno que se agrava com a criação da União Francesa, último suspiro da França colonial. Porém, é ainda clara a negligência sobre o papel da raça nos debates em torno da radicalizaçáo e continuidades no entre-guerras e de 1939 a 1945, bem como nos processos ideológicos que acompanharam a última vaga colonizadora, para uns, ou o início da descolonizaçâo para outros.

\footnotetext{
${ }^{21}$ WINTER, Jay. "The Great War in Transnational Perspective”, op. cit.

${ }^{22}$ JERÓNIMO, Miguel Bandeira. Jay Winter: A Grande Guerra foi uma guerra contra civis tanto quanto foi uma guerra entre exércitos. Lisboa: Público, 28 jun. 2014. Disponível em: <http://www.publico.pt/culturaipsilon/noticia/jay-winter-a-grande-guerra-foi-uma-guerra-contra-civis-tanto-quanto-foi-uma-guerra-entre-exercitos-1664249>. Acesso em: 4 out. 2014.

${ }^{23}$ EVANS, Andrew D. Anthropology at War: World War I and the Science of Race in Germany. Chicago, IL: University of Chicago Press, 2010; DAS, Santanu (Ed.). Race, Empire and First World War writing. Nova York: Cambridge, 2011.

${ }^{24}$ FOGARTY, Richard S. Race and War in France: Colonial Subjects in the French Army, 1914-1918. Baltimore: Johns Hopkins University Press, 2008.
} 
Heather Jones, no seu artigo As the centenary approaches: the regeneration of the First World War historiography de 2013, oferece-nos, face à aproximação do centenário da Primeira Guerra Mundial, um trabalho exímio de síntese das principais novidades historiográficas em torno de questôes históricas centrais sobre a Primeira Guerra Mundial. ${ }^{25} \mathrm{O}$ que Jones propóe no seu artigo é discutir de que forma as principais questóes, velhas e novas, em torno da Grande Guerra ganham agora uma nova roupagem pelas abordagens interdisciplinares, comparativas e transnacionais. Porque a guerra eclodiu? Como os aliados venceram a guerra? A quem cabe a responsabilidade pelas amplas perdas? Como os homens suportaram a guerra de trincheiras? Até que ponto a sociedade civil apoiou o esforço de guerra? Sáo as questôes propostas por Jones, as mesmas que, de alguma forma, outras revisões bibliográficas, como fez Jay Winter, em Legacy of the Great War (2009), ${ }^{26}$ haviam proposto alguns anos antes. Vejamos alguns desses trabalhos mais recentes.

A primeira questão seria sobre o motivo da eclosão da guerra. Duas abordagens se destacam, denunciando a persistência de uma incursão política tradicional, apesar da gradual integração, pela abordagem cultural, da opiniáo pública ou das mentalidades dos "tomadores de decisáo". Assim, por um lado, retorna-se ao debate sobre a Crise de Julho, nomeadamente através da análise das decisões diplomáticas; por outro, mantém-se a discussão sobre as condicionantes de longo termo. A renovada pesquisa sobre a Crise emerge não só de uma aproximaçáo da história às relaçôes internacionais, mostrando de que forma a guerra seria inevitável devido a falhas no sistema internacional — crise sistémica — que apanharia de surpresa grande parte da comunidade internacional. Entre outros, é o caso dos trabalhos como The Origins of the First World War de William Mulligan (2010) e An Improbable War? The Outbreak of World War I and European Political Culture Before 1914, editada por Holger Afflerbach e David Stevenson (2007) e de Christopher Clark, The Sleepwalkers: How Europe Went to War in 1914 (2012). ${ }^{27}$ Por outro lado, uma perspectiva cultural faz a sua definitiva entrada no campo das decisóes políticas no sentido de percepcionar o papel da opiniáo pública e das mentalidades políticas nos processos de decisão que levariam à guerra, veja-se o pioneirismo de Ute Frevert (2013), no âmbito da história das emoçôes, examinando como pressupostos de masculinidade e honra impediram os Estados de voltar atrás durante a Crise de Julho. ${ }^{28}$

\footnotetext{
${ }^{25}$ JONES, Heather. As the centenary approaches: the regeneration of the First World War historiography. The Historical Journal, v. 56, p. 857-878, 2013.

${ }^{26}$ WINTER, Jay (Ed.). The Legacy of the Great War: Ninety Years On, op. cit.

${ }^{27}$ MULLIGAN, William. The Origins of the First World War. New Approaches to European History Series. Cambridge: Cambridge University Press, 2010; AFFLERBACH, Holger; STEVENSON, David (Ed.). An Improbable War? The Outbreak of World War I and European Political Culture Before 1914. Nova York; Oxford: Berghahn, 2007; CLARK, Christopher, The Sleepwalkers: How Europe Went to War in 1914. Londres: Harper, 2012.

${ }^{28}$ Outras abordagens culturais de "velhos" temas devem-se aos trabalhos de RÜGER, Jan. The Great Naval Game: Britain and Germany in the Age of Empire. Cambridge: Cambridge University Press, 2009; FREVERT, Ute. Honor, gender, and power: the politics of satisfaction in pre-war Europe. In: AFFLERBACH; STEVENSON (Ed.). An Improbable War. Nova York; Oxford: Berghahn, 2007. p. 233-255.
} 
Não obstante a historiografia ainda não conseguir dar uma resposta fechada à razão por detrás da vitória dos aliados na guerra, trata-se de um tema — central — onde a história política e militar se mantém dominante. Exemplo disso é o caso de David Stevenson, em With Our Backs to the Wall: Victory and Defeat in 1918 (2011), ${ }^{29}$ que aponta que o resultado da guerra seria condicionado por questóes de logística, nomeadamente a diminuição de mantimentos na Alemanha; o colapso da Bulgária, que abriu o caminho para que os aliados libertassem os campos petrolíferos romenos, fonte crucial de combustível para os alemães, levando ao colapso nervoso temporário de Ludendorff; ou o exemplo dos historiadores britânicos, como Gary Sheffield (2001) e Jonathan Boff (2012), que defendem a ideia da "learning curve", entendida como uma importante capacidade de aprendizagem do exército britânico face ao prolongar-se da experiência de guerra e o seu especial contributo para a vitória. ${ }^{30}$

Mais interessante seria a abordagem transnacional, seja de William Philpott, Bloody Victory: the Sacrifice on the Somme (2009), seja de Elizabeth Greenhalgh em Victory Through coalition: Britain and France during the First World War (2005). O primeiro trabalho afirmando que, não obstante uma importante e difícil "vitória" aliada na Batalha do Somme, esta seria determinante para a vitória aliada, o segundo ressalvando que a vitória decorre, apesar da inicial resistência, da crescente colaboração entre aliados. ${ }^{31}$

Bem mais comum do que a tese da "learning curve" do mundo anglo-saxônico, segundo a qual os aliados ganharam a guerra por um melhoramento nas suas táticas militares, coligação, coordenação e estratégia, que implica reconhecer que os generais desempenharam um papel importante neste sucesso, nos casos francês e alemão são mais raras as análises das lideranças, muito devido à crise da historiografia militar nos anos 1960. São exceçóes os trabalhos de Elizabeth Greenhalgh, sobre Foch (2011), ${ }^{32}$ e Anna von der Goltz, sobre Hindenburg (2009). Goltz analisa — influenciada pelas abordagens culturais que retiram o foco das personalidades para se fixar na forma como estas foram apresentadas nos campos de batalha, na opinião pública e na mídia — de que maneira Hindenburg emergiu da guerra como o "símbolo heroico de um irresoluto estoicismo patriótico face ao infortúnio da Alemanha”. Apresenta-o como um mito plástico ao serviço da direita alemá, contribuindo para a compreensão da cultura política de Weimer e da ascensão nazi. ${ }^{33}$

\footnotetext{
${ }^{29}$ STEVENSON, David. With our Backs to the Wall: Victory and Defeat in 1918. Cambridge: Belknap Press of Harvard University Press, 2011.

${ }^{30}$ JONES, Heather. As the centenary approaches: the regeneration of the First World War historiography. The Historical Journal, v. 56, p. 862, 2013.

${ }^{31}$ PHILPOTT, William. Bloody Victory: the Sacrifice on the Somme. Londres: Little, Brown, 2009; GREENHALGH, Elizabeth. Victory Through Coalition: Britain and France During the First World War. Cambridge; Nova York: Cambridge University Press, 2005.

${ }^{32}$ GREENHALGH, Elizabeth. Foch in Command: the Forging of a First World War General. Cambridge: Cambridge University Press, 2011.

${ }^{33}$ GOLTZ, Anna von der. Hindenburg: Power, Myth, and the Rise of the Nazis. Oxford: Oxford University Press, 2009.
} 
A forma como os homens deram conta da guerra de trincheiras tem sido um tema largamente desenvolvido pela perspectiva cultural, interdisciplinar e transnacional. Talvez aquele que mais se beneficiou dessas abordagens. Durante muito tempo ignorados ou marginalizados, os entrincheirados vão sendo gradualmente chamados a participar da literatura em torno da guerra. As comemoraçóes dos oitenta anos do Armistício levaram a repensar a guerra. Abandona-se a tradicional perspectiva estratégica por uma abordagem política e social, primeiro no mundo anglo-saxônico. A abertura aos combatentes e às suas histórias é, assim, inaugurada por Paul Fussell (1975), John Keegan (1976) e Eric Leed (1979), que trabalham a identidade deste grupo entre o vivido, as representaçóes e os sentimentos. A evolução posterior será feita em torno do seu universo mental com Joanna Bourke (1996), Stéphane Audoin-Rouzeau (1992) ou Annette Becker (1994). Este universo de nomes torna-se ainda mais abrangente no conjunto das incursóes na cultura de guerra, sendo que os primeiros autores foram mesmo responsáveis por produzir a importante transição teórica entre uma incursão social e aquela cultural. ${ }^{34}$

Nesta linha, os primeiros trabalhos vão ao encontro da dimensão mobilizadora da fé. Incursóes neste sentido já haviam sido desenvolvidas na década de 1990 por Annette Becker, La guerre et la foi: de la mort à la mémoire, 1914-1930, onde ressalta o papel determinante da religião na mobilização quer dos soldados, quer da população, desconstruindo assim o acento na natureza secular das sociedades europeias que a história social dos anos 1960 havia consistentemente sobrevalorizado. ${ }^{35}$ Alexander Watson (2008) revela, numa ampla análise comparativa, que a resistência dos soldados na frente está associada a uma multiplicidade díspar de fatores como medo, obediência, camaradagem e que, de alguma forma, são transversais aos exércitos envolvidos na guerra. ${ }^{36}$

Sobre o universo da experiência direta dos soldados, outra dimensão explorada tem sido a da revolta e da deserção, desconstruindo-se a ideia de que seria a severa disciplina que manteria os soldados na frente da guerra. Não sendo constante, nem persistente, o que mantinha

\footnotetext{
${ }^{34}$ BECKER, Annette; AUDOIN-ROUZEAU, Stéphane. 14-18 Retrouver la guerre, op. cit., p. 34; BOURKE, Joanna. Dismembering the Male: Men' Bodies, Britain, and the Great War. Londres: Reaktion Press and Chicago; University of Chicago Press, 1996; BECKER, Annette. La guerre et la foi: De la mort à la mémoire, 1914-1930, op. cit.

35 Sobre este assunto entre outros, vejam-se SHEFFIELD, Gary. Forgotten Victory: the First World War: Myths and Realities. Londres: Headline Review, 2001; BOFF, Jonathan. Winning and Losing on the Western Front: the British Third Army and the Defeat of Germany in 1918. Cambridge, Nova York: Cambridge University Press, 2012; GREGORY, Adrian. The Last Great War: British Society and the First World War. Cambridge: Cambridge University Press, 2008. p. 152-186; HOULIHA, Patrick J. Clergy in the trenches: Catholic military chaplains of Germany and Austria-Hungary during the First World War. Chicago, IL, 2011; SNAPE, Michael; MADIGAN, Edward. The Clergy in Khaki: new perspectives on British Army chaplaincy in the First World War. Farnham; Surrey: Ashgate, 2013. MADIGAN, Edward. Faith under Fire: Anglican Army Chaplains and the Great War. Basingstoke; Nova York: Palgrave Macmillan, 2011.

${ }^{36}$ WATSON, Alexander. Enduring the Great War: Combat, Morale and Collapse in the German and British Armies, 1914-1918. Cambridge; Nova York: Cambridge University Press, 2008.
} 
os soldados à frente seria uma variedade de elementos como códigos disciplinares, culturais, etc. Tratando-se de um tema menos abordado pela historiografia britânica face à ausência de um inicial recrutamento obrigatório, o mesmo não aconteceu na Alemanha e na França. Tanto Leonard V. Smit (1994) quanto Emmanuel Saint-Fuscien (2011) ${ }^{37}$ mostram como a disciplina francesa, apesar de extremamente rigorosa em 1914, amoleceria conforme a guerra ia passando, dada a dura realidade das trincheiras; ${ }^{38}$ enquanto Joshua Sanborn (2003) ${ }^{39}$ afirma que esta não seria a razão para manter os soldados analfabetos russos na frente, mostrando que de fato havia um sentimento de patriotismo entre os soldados camponeses russos e que os motivaria a lutar. ${ }^{40}$

Retoma-se ainda o velho tema sobre o envolvimento da sociedade civil, de que forma ela aceitaria ou endossaria o esforço de guerra. Embora tivessem já passado quarenta anos desde que Jean-Jacques Becker explorou, em 1977, a reação da sociedade francesa à guerra, vendo-a como um confronto inevitável, este mantém-se como um dos campos onde mais se trabalha numa gradual substituição da ideia de um "entusiasmo" da população por uma confusão de emoções e reações tentando gerir da melhor forma aquele contexto.

Jeffrey Verhey (2000) e Roger Chickering (2007) descontroem a ideia de um amplo entusiasmo da sociedade alemã pela guerra, alegando que este seria circunscrito a jovens urbanos de classe média, particularmente estudantes. ${ }^{41}$ Adrian Gregory (2003) revela ainda que, na Grã-Bretanha, várias manifestações foram verificadas contra a mobilização quando da declaração de guerra, particularmente organizadas por sindicatos ou grupos religiosos não conformistas ${ }^{42}$. Pesquisas de Joelle Beurier (2008), Nicolas Beaupré (2006) e de Emannuelle Cronier (2012) mostram que os cidadãos franceses estariam bem informados do que se passava nas trincheiras, por uma censura deficiente e pelo contato com os soldados de

${ }^{37}$ SAINT-FUSCIEN, Emmanuel. À vos orders ? La relation d'autorité dans l'armée française de la Grande Guerre. Paris: Éditions de l'EHESS, 2011; SMITH, Leonard V. Between Mutiny and Obedience: the Case of the French Fifth Infantry Division During World War I. Princeton: Princeton University Press, 2014.

${ }^{38}$ LEMBRÉ, S.; SAINT-FUSCIEN, E. À vos ordres ? La relation d'autorité dans l'armée française de la Grande Guerre. Lectures. Les comptes rendus, 2012, mis en ligne le 15 janvier 2012. Disponível em: <htttp:// lectures.revues.org/7222>. Acesso em: 29 set. 2014.

39 SANBORN, Joshua. Drafting the Russian Nation: Military Conscription, Total War, and Mass Politics, 1905-1925. DeKalb: Northern Illinois University Press, 2003.

${ }^{40}$ Ibid, 2002. Resenha de: HOSKING, Geoffrey. (review n. 343). Disponível em: <http://www.history. ac.uk/reviews/review/343>. Acesso em: 28 set. 2014.

${ }^{41}$ CHICKERING, Roger. "War enthusiasm?" Public opinion and the outbreak of war in 1914. In: CHICKERING, Roger. An Improbable War. Oxford; Nova York: Berghahn, 2007. p. 210; ZIEMANN, Benjamin. War Experiences in Rural Germany, 1914-1923. Oxford; New York: Berg, 2007; VERHEY, Jeffrey. The Spirit of 1914: Militarism, Myth and Mobilization in Germany. Cambridge: Cambridge University Press, 2000.

${ }^{42}$ BECKER, Jean-Jacques. 1914: Comment les français sont entrés dans la guerre: contribution à l'étude de l'opinion publique printemps-été 1914, op. cit.; VERHEY, J. The Spirit of 1914: Militarism, Myth and Mobilization in Germany, op. cit.; GREGORY, Adrian. British "War Enthusiasm" in 1914: a reassessment. In: GREGORY, Adrian. Evidence, History and the Great War: Historians and the Impact of 1914-1918. Nova York: Berghahn, 2004. p. 67-85. 
licença. ${ }^{43}$ Catriona Pennell, no seu trabalho A Kingdom United (2012), expóe múltiplas reaçôes à guerra, nomeadamente como os nacionalistas irlandeses estavam dispostos a lutar pela liberdade de pequenas naçôes de forma a que tal luta os pudesse um dia ajudar. Outra questão, ainda mais intrigante, é a razão que levou a classe trabalhadora a se juntar ao esforço de guerra num momento em que o internacionalismo propagado contrariava a ordem do Estado-nação. David Silbey, em The British Working Class and Enthusiasm for War, 19141916 (2005), enfatiza o patriotismo difuso que permeava a cultura da classe trabalhadora ultrapassando a identidade nacional a fidelidade a uma identidade de classe. ${ }^{44}$

Muito além dessa escolha "voluntária" por um maior ou menor envolvimento na causa da guerra, tem sido a forma como involuntariamente todos — sem exceção — foram afetados pela guerra. Como diria Jay Winter, o "trauma é democrático, escolhe todos os tipos de pessoas na sua devastadora passagem. A história do estresse pós-traumático, configurado corretamente, não é a história da corporaçâo, mas a história da própria guerra" ${ }^{45}$ A Primeira Guerra Mundial configura-se assim como uma guerra total. Um tipo ideal interpretativo ou dispositivo heurístico usado por Roger Chickering e Stig Forster em The shadows of Total War (2009), que integra todas as dimensôes do conflito e o projeta como total, seja pela radicalizaçáo dos objetivos, pela criação de exércitos de massa, pelos métodos de guerra, pela mobilização das sociedades, pelo controle total, ou pela democratização da guerra e que se revela na completa ausência de limites espaciais e temporais da guerra. ${ }^{46}$ Nesse sentido, alguns historiadores têm mesmo sido tentados a ver o período 1914-1945 como um todo, como uma "guerra civil europeia", expressão usada redutivamente por Ernst Nolte para denotar uma luta constante entre as forças do comunismo e do fascismo. O conceito é enganoso, mas, talvez, segundo Enzo Traverso (que contraria a ideia revisionista de Nolte) lhe possa ser dada validade como um termo geral "para tentar captar o sentido de uma era de guerras e revoluçôes em que a simbiose entre cultura, política e violência profundamente moldaram as mentalidades, ideias, representaçôes e práticas de seus atores”.47

${ }^{43}$ BEAUPRÉ, Nicolas. Écrire en guerre, écrire la guerre: France, Allemagne, 1914-1919. Paris: CNRS, 2006; BEURIER, Joëlle. Information, censorship or propaganda? The illustrated French press in the First World War. In: BEURIER, Joëlle. Untold war: new perspectives in First World War studies. Boston; Leide: Brill, 2008. p. 293-324; CRONIER, Emmanuelle. L'échappée belle: permissions et permissionnaires du front à Paris pendant la Première Guerre Mondiale. Paris: Belin, 2012. PENNELL, Catriona. A Kingdom United: Popular Responses to the Outbreak of the First World War in Britain and Ireland. Oxford: Oxford University Press, 2012.

${ }^{44}$ SILBEY, David. The British Working Class and Enthusiasm for War, 1914-1916. Londres; Nova York: Frank Cass, 2005.

${ }^{45}$ WINTER, Jay. Shell-Shock and the Cultural History of the Great War. Journal of Contemporary History, v. 35, n. 1, p. 11, 2000: "Trauma is democratic; it chooses all kinds of people in its crippling passage. The history of shell-shock, properly configured, is not the history of the officer corps, but the history of the war itself" (tradução da autora).

${ }^{46}$ CHICKERING, Roger; FORSTER, Stig (Ed.). The Shadows of Total War. United Kingdom. Cambridge: Cambridge University Press, 2009.

47 TRAVERSO, Enzo. A feu et à sang. De la guerre civile européenne 1914-1945. Paris: Stock, 2007. p. 9; 
Trata-se do definitivo derrubar de barreiras entre frente e retaguarda. Assim, as sociedades do pós-guerra, sem exceção, foram marcadas por um temor da violência — um medo de que a brutalidade da guerra fosse transferida para a sociedade, em contextos de agravada crise econômica, política e social, ou mesmo, da "masculinidade" 48 — encarnada naqueles que nela tiveram maior empenho: os soldados. A nação estava assombrada "pelo medo que os antigos combatentes, o público em geral, o Estado, ou talvez os três, tivessem sido irreversivelmente 'brutalizados' pela carnificina massificada de quase quatro anos e meio de guerra”. ${ }^{4}$

Uma mobilização global que determinou transformaçôes de foro material e imaterial que ainda hoje persistem, que ainda hoje influem no atraso da percepção e compreensão da guerra. $\mathrm{O}$ desaparecimento daqueles que experimentaram diretamente a guerra, a abertura dos arquivos e a valorização do trabalho historiográfico permitiria, finalmente, um alargamento de perspectivas de análise, até à data intocáveis, sendo a morte o maior desses tabus.

As manifestações contínuas de profunda violência que se verificaram depois da Grande Guerra estariam associadas a esta e ao mito da experiência de guerra: à forma como a vivência da guerra e, acima de tudo, da morte, foram apropriadas e serviriam à brutalização da sociedade e da política. ${ }^{50}$ Retenhamos a sua definição operatória colocada por George Mosse:

Este mito resume alguns dos temas que moveram os homens numa ou noutra fase da guerra: o espírito de 1914, a guerra como um teste à masculinidade, a ideia de camaradagem e o culto dos soldados caídos - uma série de atitudes que ajudaram o homem a confrontar-se e a aceitar esta experiência sem precedentes e moldou muitas das percepçóes literárias, artísticas e políticas depois da Primeira Guerra Mundial. Seja qual for a reformulação da Europa depois da guerra, o mito da experiência de guerra torna-se um poderoso motor de vida pública e pessoal, mais nas nações insatisfeitas do que nas satisfeitas, apesar de, mesmo aí, ter que desempenhar o seu papel.51

NOLTE, Ernst. La guerre civile européenne 1917-1945: national-socialisme et bolchévisme. Paris: Syrtes, 2000. ${ }^{48}$ MOSSE, George L. Fallen Soldiers: Reshaping the Memory of the World Wars. Oxford: Oxford University Press, 1990; THEWELEIT, Klaus. Male Fantasies. v. 2: Male Bodies: Psychoanalyzing the White Terror. St. Paul: University of Minnesota Press, 1989; FORGACS, David. Fascism violence and modernity. In The Violent Muse: Violence and the Artistic Imagination in Europe, 1910-1939. Manchester: Manchester University Press, 1994, p. 5-21.

${ }^{49}$ LAWRENCE, John. Forging a peaceable kingdom: war, violence, and fear of brutalization in post-First World War Britain. The Journal of Modern History, n. 75, p. 557, 2003: "Britain after the First World War was a nation haunted by the fear that violence had slipped its chains-by the fear that the ex-servicemen, the general public, the state, or perhaps all three, had been irrevocably 'brutalized' by the mass carnage of four and a half years of war. It seemed all too easy to find evidence of 'brutalization' almost anywhere one looked" (tradução da autora).

${ }^{50}$ ISNENGHI, Mario. Il mito della Grande Guerra: da Marinetti a Malaparte. Bari: Laterza, 1970; MOSSE, George L. Le guerre mondiali dalla tragedia al mito dei caduti, op. cit.; MOSSE, George L. Fallen Soldiers. Reshaping the Memory of the World Wars, op. cit. O segundo autor influenciaria historiadores franceses como Annette Becker e Audoin-Rouzeau que procuram explicar a violência pela cultura de guerra (origem e prolongamento).

${ }^{51}$ MOSSE, George L. Two World Wars and the Myth of the War Experience. Journal of Contemporary 
$\mathrm{Na}$ sequência do trabalho de Mosse que estabelece uma relação direta entre experiência de guerra e brutalização da sociedade e política no pós-guerra, apareceu um grupo de propostas que contraria a teoria da violência. Antoine Prost e Jay Winter apresentam, no cruzamento de um conjunto de estudos desenvolvidos desde a década 1970, uma alternativa à compreensão dos antigos combatentes e dos memoriais, considerando a multiplicidade de posiçóes dos combatentes sobre a guerra e as suas comemoraçôes. Ainda na pegada de Mosse, para quem a maciça presença e integraçáo da morte estaria na base da brutalização e dos radicalismos políticos do pós-guerra, Annette Becker e Audoin-Rouzeau (2000) acrescentam que, antes mesmo do embrutecimento derivado do conflito, seria a própria cultura de guerra que induziria a esta violência extrema e não o inverso..$^{52}$ Os processos de mobilização anteriores à guerra são marcados por uma forte "bestialização" do inimigo, reduzindo-o a uma "abstração animalesca" que a guerra moderna exacerba.

A evolução da história cultural da guerra traz o significado, a assimilação e a representação da morte para o centro da análise e alarga os estudos dos fenômenos rememorativos, de uma forma geral, da guerra. ${ }^{53} \mathrm{~A}$ experiência fundamental da guerra moderna é "o assassinato de massa sancionado pelo estado". ${ }^{4} \mathrm{O}$ arrastamento do conflito e o rastro de destruiçáo marcariam profundamente náo só os combatentes, como toda a sociedade: "nada escapa à totalizaçáo do conflito, nada escapa ao sofrimento a que estes [os combatentes] estáo ligados. A morte em primeiro lugar. Ela está inscrita no psiquismo dos combatentes, percebe-se no seu espírito. Portanto, a sua relaçáo com a morte ultrapassa o quadro da frente, porque atinge através dos círculos de luto o conjunto da sociedade europeia". 55

History, v. 21, n. 4, p. 492, 1986: "This myth summarized some of the main themes which had moved men during one or another stage of the war: the spirit of 1914, the war as a test of manliness, the ideal of camaraderie and the cult of the fallen soldier - a whole series of attitudes which helped men confront and accept this unprecedented experience, and informed many of the literary, artistic and political perceptions after the first world war. Whatever the recasting of Europe after the war, the Myth of the War Experience became a powerful engine of personal and public life, more in the dissatisfied than in the satisfied nations, though even here it was destined to play its part" (tradução da autora).

${ }^{52}$ BECKER, Annette; AUDOIN-ROUZEAU, Stéphane. 14-18 Retrouver la guerre, op. cit. p. 246: "Através de representaçóes locais e manifestaçóes nacionais [...] as palavras pronunciadas nas cerimônias, as imagens oferecidas sobre forma de inscrição e dos monumentos comemorativos, vitrais, cemitérios, ossários, perduram até aos nossos dias, permite-nos também evocar essas comemoraçôes infinitas ou liturgias políticas e de lutos privados são complementares (...) as referências a Deus, ao Estado, à propaganda, encontram-se inexoravelmente agregadas à imensa confusão" (tradução da autora).

${ }^{53}$ Cujo exemplo maior passa pelo inventário dos memoriais de guerra: PROST, Antoine. Les monuments aux morts. Culte républicain? Culte civique? Culte patriotique. In: PROST, Antoine. Les lieux de mémoire. I République. Paris: Gallimard, 1984. p. 195-225; BECKER, Annette. Les monuments aux morts: mémoire de la Grande Guerre, op. cit.; KING, Alex. Memorials of the Great War in Britain. The symbolism and Politics of Remembrance. Oxford; Nova York: Berg, 1998; INGLIS, Ken. Sacred Places: War Memorials in the Australian Landscape. Melbourne: Melbourne University Press, 1998.

${ }^{54}$ MOSSE, George L. Le guerre mondiali dalla tragedia al mito dei caduti, op. cit., p. 3.

${ }^{55}$ YPERSELE, Laurence Van (Dir.). Questions d'histoire contemporaine: conflits, mémoires et identités. Paris: PUF, 2006. p. 139: "Nul n'échappe à la totalisation du conflit, nul n'échappe aux souffrances qui y sont 
A duração e as consequências do conflito exigiram a criação de razóes de nova ordem - a guerra passa a ser em nome da pátria contra o inimigo da humanidade, a verdadeira cruzada que combina elementos sagrados e liçóes de cidadania. Segundo Mosse, um processo de naturalização, trivialização e santificação transformaram a morte de massa aceitável, dissimulando-a em estruturas naturais e arquitetônicas e associando-a a sentimentos religiosos num tipo de serviço cívico. Antoine Prost discorda da ideia de Mosse, uma vez que náo acredita neste processo, mas defende que a naturalização das sepulturas e a santificação dos mortos não significa uma indiferença, mas uma forma de lidar com emoçôes tão fortes. ${ }^{56}$

Mais recentemente a discussão sobre radicalização tem-se desenvolvido em torno do Genocídio Armênio, que, longamente ignorado, tem sido visto como evento-chave da Grande Guerra. ${ }^{57}$ Maud Mandel (2003) revela, numa comparaçáo com o Holocausto, o quanto a negligência do estudo do Genocídio Armênio está associada à existência de um Estado-nação que suporte ou reivindique o seu reconhecimento histórico e memorial. ${ }^{58}$ Jay Winter vê no Genocídio Armênio o auge da radicalização da violência e acrescenta que "o genocídio aconteceu em ambas as guerras mundiais porque o assassínio de civis na defesa do teu país tornou-se normal e legítimo. Esses são os fatos cruciais que sustentam o argumento de que houve uma degeneração da condução da guerra em 1914-1918, que criou condiçôes para catástrofes posteriores". 59

O dualismo sobre a existência, ou não, de uma relação entre a guerra e a brutalização política da sociedade no pós-guerra persiste nas abordagens mais recentes à guerra. As determinaçóes nacionais sobre os estudos revelam como se deve atender a cada caso com o devido cuidado de não estabelecer interpretaçôes generalizantes.

liées. À la mort en premier lieu. Elle est inscrite dans le psychisme des combattants, voir dans leur chair. Cependant, ce rapport à la mort dépasse le cadre du front, puisqu'il atteint par les cercles de deuil l'ensemble de la société européenne. (...) Les deuils ont, en outre, eu bien des difficultés à se faire, du fait souvent de l'absence des corps du disparu dont il s’agit alors de perpétuer la mémoire" (tradução da autora).

${ }^{56}$ PROST, Antoine. The Impact of War on French and German Political Cultures. The Historical Journal, v. 37, n. 1, p. 209, 1994.

${ }^{57}$ Muitos trabalhos têm sido desenvolvidos sobre o assunto, isto é, deportação de populaçóes inteiras: GATRELL, Peter. A Whole Empire Walking: refugees in Russia during World War I. Bloomington, 1999; LOHR, Eric. Nationalizing the Russian empire: the Campaign Against Enemy Aliens During World War I. Cambridge, MA; Londres: Harvard University Press, 2003.

${ }^{58}$ MANDEL, Maud S. In the Aftermath of Genocide: Armenians and Jews in Twentieth-Century France. Durham; Londres: Duke University Press, 2003.

59 JERÓNIMO, Miguel Bandeira. Jay Winter: A Grande Guerra foi uma guerra contra civis tanto quanto foi uma guerra entre exércitos. Lisboa: Público, 28 jun. 2014. Disponível em: <http://www.publico.pt/culturaipsilon/noticia/jay-winter-a-grande-guerra-foi-uma-guerra-contra-civis-tanto-quanto-foi-uma-guerra-entre-exercitos-1664249>. Acesso em: 4 out. 2014. 


\section{Incompleto fim de fronteiras — a quarta geração}

Jay Winter e Antoine Prost apresentam uma série de argumentaçóes que solidificam a perspectiva de que a análise da guerra deve ser, fundamentalmente, nacional: a guerra é a "apoteose destrutiva da naçáo" em crise, nomeadamente dos valores do Estado-nação; a história é escrita a partir de um ponto de vista nacional em que o historiador se insere; o desenvolvimento de uma ideia de "supra-nação europeia" deita por terra os valores do voluntarismo; as periodizaçóes são diferentes. Cada cultura desenvolveu modelos narrativos nacionais, daí, por exemplo, a obra de Paul Fussell não ter sido traduzida em francês, porque a abordagem da guerra como ironia náo é perceptível nesse contexto. A memória construída sobre a experiência da guerra é historicamente muito diferente, não pode ser pensada como guerra europeia a partir de quadros nacionalmente estruturados. ${ }^{60}$

A construção da memória da Primeira Guerra Mundial está intimamente ligada à experiência nacional de guerra, mas também a um conjunto de representaçôes herdadas e que garantem a manutenção e persistência identitária, servindo ou não à legitimação de memória oficialmente delineada. ${ }^{61}$ Fenômenos que reemergem no imediato pós-guerra, numa tentativa da persistência mítica das unióes sagradas, evidenciando, porém, a incapacidade de recuperar os ideais propagados no início do conflito, face a uma experiência de guerra que marcaria profundamente aqueles que direta ou indiretamente dela participaram.

Porém, principalmente Jay Winter, não obstante apregoar à partida os condicionamentos nacionais que determinam a abordagem historiográfica, desconstruiria esta mesma visão num conjunto de trabalhos que viria a publicar.

Inicialmente, aparece a possibilidade de se realizar uma história comparativa com o Historial de Péronne e com estudos localizados sobre naçôes vizinhas, como é o caso de Capital Cities at War: Paris, London, Berlin, 1914-1919.62 Depois, muito concretamente, Winter chama atenção, sendo aliás tema central da conferência que assinalou, em Londres, o centenário da Grande Guerra, de que desde há algum tempo se configura uma quarta geração historiográfica, denominada de transnational generation (geração transnacional).

Importa distinguir a abordagem internacional da transnacional. Por mais de um século, a Primeira Guerra Mundial foi entendida em termos das relaçóes internacionais, onde níveis de colaboração e confronto nacional e imperial tomavam parte.

Beneficiando profundamente de seus predecessores, a história transnacional assume muitos níveis para lá da experiência nacional. Por exemplo, a história da deserção que acon-

\footnotetext{
${ }^{60}$ Também porque as tradiçôes culturais diferem: "ironia britânica", "cartesianismo francês" e "sociologia weberiana”. Veja-se sobre isto WINTER, Jay; PROST, Antoine. Penser la Grande Guerre : Un essai d'historiographie. Paris: Éditions du Seuil, 2004, p. 264-270.

${ }^{61}$ LEMOINE, Thierry. 'Culture(s) de guerre', évolution d'un concept, op. cit., p. 146.

${ }^{62}$ WINTER, Jay; ROBERT, Jean-Louis. Capital cities at war: Paris, Londres, Berlin 1914-1919. Cambridge: Cambridge University Press, 1997.
} 
teceu em muitos exércitos para além de modelos nacionais, sendo as razóes que estiveram na sua origem transversais. Enfim, o potencial encarnado na abordagem transnacional é evidente numa instituição claramente comprometida em escapar dos confins de uma história nacional da guerra: Historial de la Grande Guerre - Péronne (França) — um museu e centro de pesquisa da Grande Guerra, resultante da colaboração entre Alemanha, França e Grã-Bretanha. Foi desenhado por historiadores e escrito em três línguas, colocado no centro de comando do exército alemão durante a batalha do Somme, onde Ernst Junger considerou ser o local onde nasceu o século XX. Juntos, os historiadores criaram um museu transnacional: para entender a integração da Europa nos anos 1990, tinha-se que entender a sua desintegração no início do século. ${ }^{63}$

Este trabalho decorre, no entanto, de um esforço de várias geraçóes de historiadores que os precederam, sendo a historiografia da Grande Guerra cumulativa e multifacetada. Nenhum historiador social ou cultural ignora a história da nação, mas a guerra é um acontecimento tão marcante que vai muito além das fronteiras materiais ou imateriais da nação. Jay Winter, coordenador da Cambridge History of the First World War (2013), a última grande coleção de textos sobre a Primeira Guerra Mundial, refere que ao "adotar uma abordagem estritamente comparativa, evitamos as limitaçóes das histórias nacionais, resultantes das narrativas nacionais já existentes e predominantes. (...) Em suma, providenciamos um método para escrever a história da guerra que extravasou as fronteiras nacionais de cada país interveniente". ${ }^{64}$

Não comportando esta revisão historiográfica textos produzidos no ano de 2014, quando se tem constatado uma proliferação, quase que massiva, de publicaçóes em torno da guerra, o que anunciam alguns projetos europeus de investigação é uma forte colaboração transnacional, com a criação de plataformas on-line em torno das múltiplas experiências da guerra. Parece que, finalmente, a academia saiu das fronteiras do nacional. ${ }^{65}$

Porém, não obstante o desejo de se fazer uma história transnacional do conflito, a rememoração dos cem anos do início da Primeira Guerra Mundial, neste ano de 2014, vem mais uma vez denunciar que aquilo que se configurou como uma das principais causas do conflito - o confronto de nações - persiste nas políticas oficiais de memória, face às quais a historiografia não está ilesa de responsabilidades. As galerias da Primeira Guerra Mundial no Imperial War Museum são a face dessa dimensão nacional que à guerra levaria, pela quase total exclusão, numa exposição oficial, de referências aos aliados e aos ini-

${ }^{63}$ WINTER, Jay (Ed.). The Legacy of the Great War: Ninety Years On, op. cit., p. 9.

${ }^{64}$ JERÓNIMO, Miguel Bandeira. Jay Winter: A Grande Guerra foi uma guerra contra civis tanto quanto foi uma guerra entre exércitos. Lisboa: Público, 28 jun. 2014. Disponível em: <http://www.publico.pt/culturaipsilon/noticia/jay-winter-a-grande-guerra-foi-uma-guerra-contra-civis-tanto-quanto-foi-uma-guerra-entre-exercitos-1664249>. Acesso em: 4 de out. 2014.

${ }^{65}$ Para além de plataformas de universidades, bibliotecas, museus e arquivos nacionais, foi criada, a nível europeu, a mais significante plataforma de cruzamento de informação sobre a Primeira Guerra Mundial, a Europeanna. Disponível em: <http://www.europeana1914-1918.eu/en>. Acesso em: 4 out. 2014. 
migos, mas mais gravemente àqueles que, integrando o império britânico, não são dignos de ocupar as galerias do Museu Imperial Britânico, sejam eles australianos, indianos ou sul-africanos!

\section{Referências bibliográficas}

AFFLERBACH, Holger; STEVENSON, David (Ed.). An Improbable War? The Outbreak of World War I and European Political Culture Before 1914. Nova York; Oxford: Berghahn, 2007.

AUDOIN-ROUZEAU, Stéphane. Préface. In: De la Grande Guerre au totalitarisme. La brutalisation des sociétés européenes. Paris: Hachete Littératures, 1999.

BEAUPRÉ, Nicolas. Écrire en guerre, écrire la guerre: France, Allemagne, 1914-1919. Paris: CNRS, 2006.

BECKER, Annette. La guerre et la foi: De la mort à la mémoire, 1914-1930. Paris: A. Colin, 1994.

. Les monuments aux morts: mémoire de la Grande Guerre. Paris: Éditions Errance, 1991.

. War and Faith: the religious imagination in France, 1914-1930. Oxford; Nova York: Berg, 1998.

BECKER, Annette; AUDOIN-ROUZEAU, Stéphane. 14-18 Retrouver la guerre. Paris: Gallimard, 2000.

BECKER, Jean-Jacques. 1914: Comment les français sont entrés dans la guerre: contribution à l'étude de l'opinion publique printemps-été 1914. Paris: Presses de la Fondation Nationale des Sciences Politiques, 1977.

BEURIER, Joëlle. Information, censorship or propaganda? The illustrated French press in the First World War. In: BEURIER, Joëlle. Untold war: new perspectives in First World War studies. Boston; Leide: Brill, 2008.

BOFF, Jonathan. Winning and Losing on the Western Front: the British Third Army and the Defeat of Germany in 1918. Cambridge, Nova York: Cambridge University Press, 2012.

BOURKE, Joanna. Dismembering the Male: Men' Bodies, Britain, and the Great War. Londres: Reaktion Press and Chicago; University of Chicago Press, 1996.

CHICKERING, Roger. "War enthusiasm?" Public opinion and the outbreak of war in 1914. In: CHICKERING, Roger. An Improbable War. Oxford; Nova York: Berghahn, 2007. CHICKERING, Roger; FORSTER, Stig (Ed.). The Shadows of Total War. United Kingdom. Cambridge: Cambridge University Press, 2009. 
CLARK, Christopher, The Sleepwalkers: How Europe Went to War in 1914. Londres: Harper, 2012.

CRONIER, Emmanuelle. L'échappée belle: permissions et permissionnaires du front à Paris pendant la Première Guerre Mondiale. Paris: Belin, 2012.

DAS, Santanu (Ed.). Race, Empire and First World War writing. Nova York: Cambridge, 2011. EKSTEINS, Modris. Rites of spring: the Great War and the birth of modern age. Boston: Houghton, 1989.

EVANS, Andrew D. Anthropology at War: World War I and the Science of Race in Germany. Chicago: University of Chicago Press, 2010.

FOGARTY, Richard S. Race and War in France: Colonial Subjects in the French Army, 1914-1918. Baltimore: Johns Hopkins University Press, 2008.

FORGACS, David. Fascism violence and modernity. In: The Violent Muse: Violence and the Artistic Imagination in Europe, 1910-1939. Manchester: Manchester University Press, 1994.

FREVERT, Ute. Honor, gender, and power: the politics of satisfaction in pre-war Europe. In: AFFLERBACH, Holger; STEVENSON, David (Eds.). An Improbable War. Nova York; Oxford: Berghahn, 2007.

FUSSELL, Paul. The Great War and Modern Memory. Nova York; Londres: Oxford University Press, 1975.

GATRELL, Peter. A Whole Empire Walking: refugees in Russia during World War I. Bloomington: Indiana University Press, 1999.

GOLTZ, Anna von der. Hindenburg: Power, Myth, and the Rise of the Nazis. Oxford: Oxford University Press, 2009.

GREENHALGH, Elizabeth. Foch in Command: the Forging of a First World War General. Cambridge: Cambridge University Press, 2011.

- Victory Through Coalition: Britain and France During the First World War. Cambridge; New York: Cambridge University Press, 2005.

GREGORY, Adrian. British "War Enthusiasm" in 1914: a reassessment. In: GREGORY, Adrian. Evidence, History and the Great War: Historians and the Impact of 1914-1918. Nova York: Berghahn, 2004.

The Last Great War: British Society and the First World War. Cambridge: Cambridge University Press, 2008.

HORNE, John. Labour at War: France and Britain 1914-1918. Oxford: Clarendon Press, 1991.

HOULIHA, Patrick J. Clergy in the trenches: Catholic military chaplains of Germany and Austria-Hungary during the First World War. Chicago: Chicago University Press, 2011. 
HYNES, Samuel. A War Imagined. The Great War and English Culture. Londres: Bodley Head, 1991.

. The Soldier's Tale. Bearing Witness to Modern War. Nova York: Penguin Press, 1997.

INGLIS, Ken. Sacred Places: War Memorials in the Australian Landscape. Melbourne: Melbourne University Press, 1998.

ISNENGHI, Mario. Il mito della Grande Guerra: da Marinetti a Malaparte. Bari: Laterza, 1970. JOLL, James. 1914: The Unspoken Assumptions. Londres: Weidenfeld and Nicolson, 1968. JONES, Heather. As the centenary approaches: the regeneration of the First World War historiography. The Historical Journal, v. 56, p. 857-878, 2013.

KEEGAN, John. The Face of the Battle. Londres: Hutchinson, 1998.

KING, Alex. Memorials of the Great War in Britain. The symbolism and Politics of Remembrance. Oxford; Nova York: Berg, 1998.

LAWRENCE, John. Forging a peaceable kingdom: war, violence, and fear of brutalization in post-First World War Britain. The Journal of Modern History, n. 75, p. 557, 2003.

LEED, Eric J. No Man's Land: Combat and Identity in World War I. Cambridge: Cambridge University Press, 1979.

LEMOINE, Thierry. 'Culture(s) de guerre', évolution d'un concept. In: - Questions d'histoire contemporaine: conflits, mémoires et identités. Paris: PUF, 2006.

LOHR, Eric. Nationalizing the Russian Empire: the Campaign against Enemy Aliens during World War I. Cambridge, MA; Londres: Harvard University Press, 2003.

MADIGAN, Edward. Faith under Fire: Anglican Army Chaplains and the Great War. Basingstoke; Nova: Palgrave Macmillan, 2011.

MANDEL, Maud S. In the Aftermath of Genocide: Armenians and Jews in Twentieth-Century France. Durham; Londres: Duke University Press, 2003.

MOSSE, George L. Fallen Soldiers: Reshaping the Memory of the World wars. Oxford: Oxford University Press, 1990. - Le guerre mondiali dalla tragedia al mito dei caduti. Roma: Laterza, 1990 . Two World Wars and the Myth of the War Experience. Journal of Contemporary History, v. 21, n. 4, p. 492, 1986.

MULLIGAN, William. The Origins of the First World War. New Approaches to European History Series. Cambridge: Cambridge University Press, 2010.

NOLTE, Ernst. La guerre civile européenne 1917-1945: national-socialisme et bolchévisme. Paris: Syrtes, 2000.

PENNELL, Catriona. A Kingdom United: Popular Responses to the Outbreak of the First World War in Britain and Ireland. Oxford: Oxford University Press, 2012. 
PHILPOTT, William. Bloody Victory: the Sacrifice on the Somme. Londres: Little, Brown, 2009.

PROST, Antoine. 1925: Renouvin et les origines de la première guerre mondiale. Le Monde, 4 nov. 2013. Disponível em: <http://www.lemonde.fr/livres/article/2013/11/04/1925-renouvin-et-les-origines-de-la-premiere-guerre-mondiale_3507594_3260.html>. Acesso em: 14 set. 2014.

. Les anciens combattants et la société française, 1914-1939. 3 v. Paris: Presses de la FNSP, 1977.

. Les monuments aux morts. Culte républicain? Culte civique? Culte patriotique. In: PROST, Antoine. Les lieux de mémoire. I République. Paris: Gallimard, 1984.

- Republican Identities in War and Peace: Representations of France in the Nineteenth and Twentieth Centuries. Oxford: Berg, 2002.

- The Impact of War on French and German Political Cultures. The Historical Journal, v. 37, n. 1, p. 209, 1994.

RÜGER, Jan. The Great Naval Game: Britain and Germany in the Age of Empire. Cambridge: Cambridge University Press, 2009.

SAINT-FUSCIEN, Emmanuel. À vos orders ? La relation d'autorité dans l'armée française de la Grande Guerre. Paris: Éditions de l'EHESS, 2011.

SANBORN, Joshua. Drafting the Russian Nation: Military Conscription, Total War, and Mass Politics, 1905-1925. DeKalb: Northern Illinois University Press, 2003.

SHEFFIELD, Gary. Forgotten Victory: the First World War: Myths and Realities. Londres: Headline Review, 2001.

SILBEY, David. The British Working Class and Enthusiasm for War, 1914-1916. Londres e Nova York: Frank Cass, 2005.

SMITH, Leonard V. Between Mutiny and Obedience: the Case of the French Fifth Infantry Division During World War I. Princeton: Princeton University Press, 2014.

SNAPE, Michael; MADIGAN, Edward. The Clergy in Khaki: new perspectives on British Army chaplaincy in the First World War. Farnham; Surrey: Ashgate, 2013.

STEVENSON, David. With our Backs to the Wall: Victory and Defeat in 1918. Cambridge: Belknap Press of Harvard University Press, 2011.

THEWELEIT, Klaus. Male Fantasies. v. 2: Male Bodies: Psychoanalyzing the White Terror. University of Minnesota Press, 1989.

TRAVERSO, Enzo. A feu et à sang. De la guerre civile européenne 1914-1945. Paris: Stock, 2007.

VERHEY, Jeffrey. The Spirit of 1914: Militarism, Myth and Mobilization in Germany. Cambridge: Cambridge University Press, 2000. 
WATSON, Alexander. Enduring the Great War: Combat, Morale and Collapse in the German and British Armies, 1914-1918. Cambridge; Nova York: Cambridge University Press, 2008.

WINTER, Jay (Ed.). The Legacy of the Great War: Ninety Years On. Columbia; Londres: University of Missouri Press, 2009.

. "The Great War in Transnational Perspective". In: World War One International Conference. Londres: Queen Mary University of London, 1-4 de ago. 2014.

. Shell-Shock and the Cultural History of the Great War. Journal of Contemporary History, v. 35, n. 1, p. 11, 2000.

. Sites of war, sites of mourning: The Great War in the European Cultural History. Cambridge: Press Syndicate of the University of Cambridge, 1995. . Socialism and the Challenge of War. Ideas and Politics in Britain 1912-18. Londres: Routledge, 1974.

WINTER, Jay; PROST, Antoine. Penser la Grande Guerre: Un essai d'historiographie. Paris: Éditions du Seuil, 2004.

WINTER, Jay; ROBERT, Jean-Louis. Capital cities at war: Paris, London, Berlin 1914-1919. Cambridge: Cambridge University Press, 1997.

YPERSELE, Laurence Van (Dir.). Questions d'histoire contemporaine: conflits, mémoires et identités. Paris: PUF, 2006.

ZIEMANN, Benjamin. War Experiences in Rural Germany, 1914-1923. Oxford; Nova York: Berg, 2007. 